\title{
Analysis of Forensic Casework Utilizing Infrared Microspectroscopic Imaging
}

\author{
Adam Lanzarotta ${ }^{1}$ \\ 1. FDA Forensic Chemistry Center, Cincinnati, OH, USA
}

The ability to examine samples in their native state is one of the most significant advantages offered by infrared spectroscopy compared to many other analytical techniques. Examinations using this approach are generally fast, require minimal sample preparation and often do not require the use of solvents. Although it is not nearly as sensitive as chromatographic/mass spectrometric approaches, infrared spectroscopy can be conducted on a wide variety of sample types. Regarding macro infrared spectroscopy, which typically uses a single element detector with a sampling aperture on the order of $\mathrm{mm}$, many of these advantages are often limited to pure compounds or analytes present above $1 \%$ in a non-interfering matrix [1]. Multi-component samples can be separated manually and individual particles can be identified using infrared microspectroscopy, which also utilizes a single-element detector but with a smaller aperture that is closer to the size of individual particles (down to about 10 $\mu \mathrm{m})$. Unfortunately, infrared microspectroscopic analyses are often time consuming, require a skilled analyst and can be difficult when the sample contains small particles or several particle types with a similar morphology.

In many cases a more efficient and effective approach includes infrared spectroscopic imaging, which uses a multi-channel detector to collect an infrared spectrum at each spatial location in a twodimensional region of interest. As a result, particles that are physically separated from one another often yield spectra that are characteristic of nearly pure compounds, thereby providing the ability to identify individual ingredients of a multi-component sample. For example, forensic analyses using infrared spectroscopic imaging at the Forensic Chemistry Center (FCC) have included determining the composition of combination drugs (those with more than one active ingredient) [1], suspected counterfeit tablets [2], illicit pharmaceuticals [3], dietary supplements [4], human autopsy tissue extractions [5] and cross-sectioned suspected counterfeit packaging materials such as adhesive labels, foil safety seals and cigarette tear tape [6]. It is the intent of this presentation to review these cases as well as describe additional, yet to be published analyses conducted by FCC that highlight some additional advantages of this instrumental approach that has the ability to answer forensically relevant questions that cannot be readily answered, or answered as easily, using many other techniques.[7]

Currently unpublished examples include the analysis of animal tissue sections for toxic inclusions, drugs/dietary supplements, an antibiotic with an active pharmaceutical ingredient (API) present as several different salt forms, an adulterated bulk API, unknown trace powders for illicit drugs and an ophthalmic solution suspected of being adulterated with bleach. A brief description of the first example is provided in Fig. 1.[7] Fig. 1a is a photomicrograph of a cross-sectioned kidney tissue section from a cat that consumed adulterated pet food. A correlation search for melamine cyanurate yielded the image in Fig. 1b where bright regions correspond to pixels with spectra that have a high correlation to the target compound. Figs. 1a and $1 \mathrm{~b}$ have been overlaid in Fig. 1c, which indicates that the high correlation regions correspond to bright circular regions in the tissue. The representative R/A infrared spectrum from a low correlation region provided in Fig. 1d exhibited absorptions characteristic of protein. The representative R/A infrared spectrum from a high correlation region provided in Fig. 1e is clearly not consistent with that of the tissue, although its poor quality prohibited further identification. As a result, 
the stage was positioned on a high melamine-cyanurate correlation region and a "drop-down" attenuated total reflection (ATR) accessory was lowered until it made contact with the sample. The resulting ATR spectrum, Fig. 1f, exhibited a photometrically accurate spectrum that was consistent with the melamine cyanurate standard reference spectrum provided in Fig. 1g.

Based on the current literature and results of this study, infrared spectroscopic imaging has proven to be useful for examining a wide range of multi-component forensic casework samples. The results described in this presentation will not advocate that this technique be used in a vacuum; rather, it will demonstrate that imaging is useful in conjunction with other methods early in an investigation, and as a means to provide a more accurate overall assessment of a suspect product.

\section{References:}

[1] A Lanzarotta. Appl. Spectrosc. 2015, 69, 205-214.

[2] A Lanzarotta et al, Anal. Chem. 2011, 83, 5972-78.

[3] A Lanzarotta et al, Spectroscopy, 2011, 26, 34-41.

[4] A Lanzarotta et al, J. Pharm. Biomed. Anal. 2012, 67-68, 22-27.

[5] A Lanzarotta, CM Kelley. Journal of Forensic Sciences. Available online January 6, 2016. 1-8. http://onlinelibrary.wiley.com/doi/10.1111/1556-4029.13018/full.

[6] T Winner, A Lanzarotta, AJ Sommer. Submitted for publication to Microscopy and Microanalysis. October, 2015.

[7] A Lanzarotta. Submitted for publication. December, 2015.
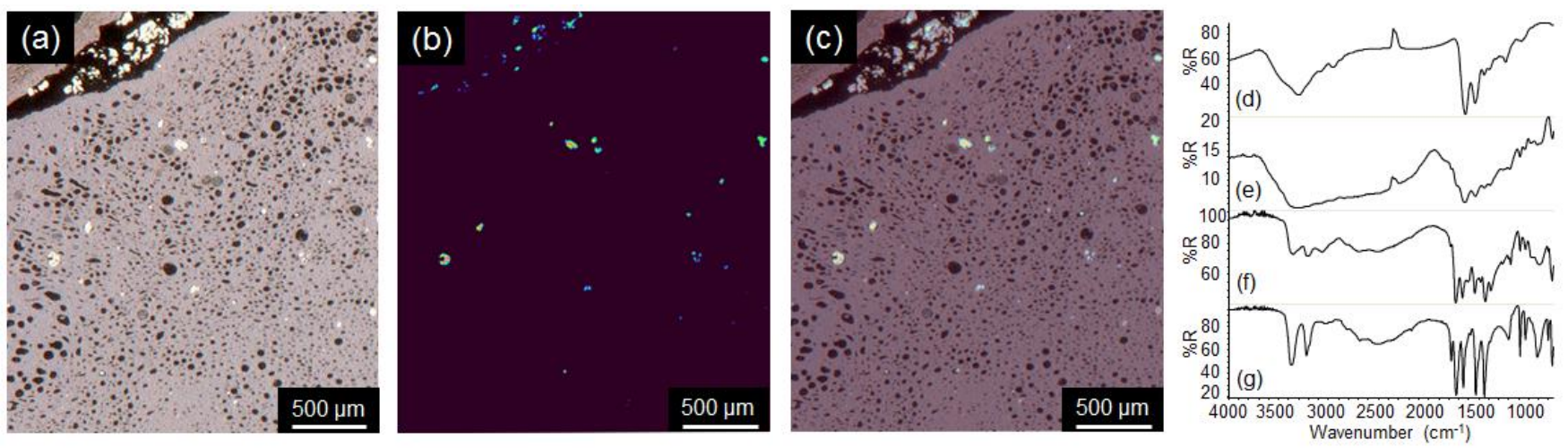

Figure 1. Visible image (a), R/A correlation image for melamine cyanurate (b), overlay image of a and b (c), representative R/A spectrum from a low correlation region (d), representative R/A spectrum from a high correlation region (e), ATR spectrum from a high correlation region (f) and ATR spectrum of a melamine cyanurate pure compound reference standard $(\mathrm{g})$. 